\title{
An effective combination of finite element and differential quadrature method for analyzing of plates partially resting on elastic foundation
}

\author{
Mehdi Dehghan, Mohammad Zamani Nejad* and Amin Moosaie
}

Mechanical Engineering Department, Yasouj University, P. O. Box: 75914-353, Yasouj, Iran

\begin{tabular}{|c|c|}
\hline$\overline{\text { A R T I C L EI N F O }}$ & A B S T RA C T \\
\hline $\begin{array}{l}\text { Article history: } \\
\text { Received } 6 \text { March, } 2016 \\
\text { Accepted } 23 \text { June } 2016 \\
\text { Available online } \\
\text { 23 June } 2016 \\
\text { Keywords: } \\
\text { FE-DQ method } \\
\text { Stability and free vibration } \\
\text { Thick plates } \\
\text { Partial elastic foundation }\end{array}$ & $\begin{array}{l}\text { This paper is concerned with the vibration and stability analysis of thick rectangular plates } \\
\text { resting on elastic foundation, which is distributed over the particular area of the plate. A two- } \\
\text { parameter (Pasternak) model is considered to describe the elastic foundation. The eigenvalue } \\
\text { problem in 3-D domain is numerically solved by a combination of the finite element and } \\
\text { differential quadrature method (DQM). The energy principle is employed to derive the } \\
\text { governing equations in the framework of three-dimensional, linear and small strain theory of } \\
\text { elasticity. The in-plane domain of the problem is discretized using two-dimensional finite } \\
\text { elements and spatial derivatives of equations in the thickness direction are discretized in strong- } \\
\text { form using DQM. As a first endeavor, the mixed FE-DQ method has been employed for 3-D } \\
\text { buckling and free vibration analysis of rectangular thick plates partially supported by an elastic } \\
\text { foundation. The accuracy of obtained results is validated by comparing to the few analytical } \\
\text { solutions in the literature. }\end{array}$ \\
\hline
\end{tabular}

(C) 2016 Growing Science Ltd. All rights reserved.

\section{Introduction}

The vibration of plates resting on elastic foundations, which has practical applications in civil, mechanical, marine and aerospace engineering, has been investigated extensively. In addition, various analytical and numerical methods have been employed to study this problem. Generally, a lot of engineering problems can be modeled as thick plates on elastic foundations such as footings and raft foundations of variety of structures, pavement of roads and bases of heavy machines. It should be noted that, the mechanical behavior of elastic foundations was widely discussed by Winkler (1867) and Pasternak (1954) (as a two-parameter model). Different two-dimensional and three-dimensional theories by numerical or analytical methods can be used to analyze the plates on elastic foundations. The two-dimensional plate theories including classical plate theory (CPT), the first order shear

* Corresponding author. Tel. \& Fax.: +98 7433221711

E-mail addresses: m.zamani.n@gmail.com m zamani@yu.ac.ir (M. Zamani Nejad) 
deformation plate theory (FSDT) and the higher order shear deformation plate theories (HSDT) are commonly used for the analysis of plates. The classical plate theory (Timoshenko and WoinowskyKrieger, 1970) assumes that the straight lines, initially normal to the mid-plane, remain straight and normal to the mid-plane during the deformation (known as Kirchhoff hypotheses). This means that the vertical shear strains are negligible. The thin plate theories are assumed in which the reaction forces of elastic foundation are acting on middle surface of plates (Leissa, 1973), whereas in thick plate analysis, the effects of elastic foundation on the upper and lower surfaces of the plates are obviously different. In first-order shear deformation theory, a constant shear strain distribution is considered through the thickness of the plates (Mindlin, 1951). A correction factor is then introduced to reduce the errors resulting from this hypothesis. The higher-order shear deformation theory is then proposed to represent better the shear stress distribution along the thickness direction. It should be mentioned that the inherent deficiency is unavoidable in these approximate theories because the transverse normal stress is not considered (Lim, 1999).

Despite the 2-D analysis, a three dimensional analysis does not rely on any assumption about kinematics of deformation of a plate. Consequently, such analyses not only provide more realistic results but also reveal physical characteristics which cannot otherwise be predicted by 2-D analysis. Takahashi and Sonoda (1992) presented results for buckling and free vibration of thin plates on elastic foundation. The free vibration and buckling analysis of rectangular Mindlin plate on elastic foundation with simply supported boundary condition was performed by Xiang et al. (1994). The finite element method was employed by Omurag et al. (1997) for free vibration analysis of thin plates on elastic foundation. Lam et al. (2000) used Green functions to study the bending, buckling and free vibration of Levy plates on elastic foundation. The higher-order shear deformation theory using power expanded series was used by Matsunaga (2000), for three-dimensional analyses of thick plates on elastic foundation. Thereafter, the vibration behavior of rectangular Mindlin plates resting on nonhomogenous elastic foundation was studied in detail (Xiang, 2003).

The Ritz method was used by Zhou et al. (2004) for free vibration analysis of thick rectangular plates based on three-dimensional theory of elasticity. The partially supported assumption regarding free vibration analysis of thin rectangular plates has been suggested by Motaghian et al. (2012). Lately, in analyzing of thin plates resting on elastic foundation, the innovative superposition method base on Hamiltonian system has been presented by Pana et al. (2013). The refined shear deformation theory has been proposed by Thai et.al (2013) for bending, buckling, and vibration of plates on elastic foundation. This theory is based on assumption that the in-plane and transverse displacements consist of bending and shear components. This theory does not require shear correction factor and has strong similarities with CPT in some aspects. Use of shear forces instead of rotational displacements has been suggested by Thai and Choi (2014) as zeroth-order shear deformation theory for bending and vibration analyses of functionally graded plates resting on elastic foundation.

Samaei et al. (2015) derived an explicit solution for obtaining the natural frequencies of the graphene sheet embedded in elastic medium using nonlocal Mindlin plate theory. The finite element method was used by Vimal et al. (2014) to study the free vibration analysis of functionally graded skew plates. Initially, Bellman et al. (1972) proposed the DQ method as a simple and rapid solution method to solve nonlinear partial differential equations. The Generalized Differential Quadrature (GDQ) method was implemented to analyze some structural problems by Du et al. (1994). Thereafter, the GDQ method has been widely used to carry out three-dimensional analyses of rectangular plates (Liew, 1998, 1999, Teo, 1999). Recently, The Differential Quadrature Method (DQM) has been used for free vibration analysis of nonhomogeneous orthotropic rectangular plate resting on elastic foundation (Gupta et al., 2016). Previously, the FE-DQ method has been used to analyze the free vibration and buckling of thick plates (Dehghan and Baradaran, 2011).

Observation from the literature listed above and to the best of author's knowledge indicates that, the 3-D buckling and free vibration analysis of thick rectangular plates partially resting on an elastic 
foundation is scarce. Lack of an efficient numerical method for analyzing the partially supported plates is the shortcoming which seems to be existed. So, in this paper a vigorous mixed method is introduced which benefits the ability of FEM in modeling of complicated geometry and boundary conditions and at the same time gains the simplicity and accuracy of DQM. The Finite Element Method (FEM) is a well-established numerical method which has been applied to boundary value problems in different fields of engineering and applied sciences. In spite of its numerous advantages, there are some drawbacks associated with the application of conventional FEM for plate analyses. The shear locking phenomenon is the main drawback of conventional FE method in 2-D analysis of plates. Although, the shear locking phenomenon does not appear in 3-D analyses, the building of a 3-D mesh of 3-D elements with suitable aspect ratio to obtain efficiently a 3-D solution of thick plates is not a trivial task. In this article, a coupled FE-DQ method is proposed for 3-D analysis of thick rectangular plates resting on elastic foundations, with various boundary conditions.

In section 2, the basic features of the DQ method are illustrated and recessive relations for approximation of a function and its derivatives in a problem domain are introduced. In part 3 the Hamilton's principle is used to derive the required equations to solve buckling and free vibration of thick plates on elastic foundations based on the mixed FE-DQ method. Finally, in section 4, the accuracy and convergence of the results obtained for different geometry and boundary conditions of rectangular plates are discussed.

\section{DQ Method}

The DQ method has two major features. Firstly, the method approximates a function on the global area using higher-order polynomials. Secondly, this method directly approximates the derivatives of a function at a point in terms of function values alone. In this method the spatial derivatives of functions are approximated by using some series including the weighting coefficients and the function values at all grid points in the solution domain of that spatial variable. This can be illustrated by considering a one-dimensional function. According to the method, the $m t h$ derivative of a function $u(z, t)$ can be approximated by:

$$
\frac{\partial^{m} u\left(z_{i}, t\right)}{\partial z^{m}}=u_{z}^{(m)}\left(z_{i}, t\right)=\sum_{j=1}^{N} A_{i j}^{(m)} u\left(z_{i}, t\right) \quad \text { For } i, j=1,2, \ldots, N \text { and } m=1,2, \ldots, N-1
$$

where $\mathrm{N}$ is the number of grid points in the spatial variable direction. The term $A_{i j}^{(m)}$ in the previous equation is weighting coefficients at $i t h$ points of solution domain. According to the DQ method, the weighting coefficients can be obtained using the following recurrence formula ( $\mathrm{Du}, 1994)$ :

$$
\begin{array}{ll}
A_{i j}^{(m)}=m\left(A_{(i j)}^{(m-1)} A_{i j}^{1}-\frac{A_{i j}^{(m-1)}}{z_{i}-z_{j}}\right) & \text { For } i \neq j, m=2,3, \ldots, N-1, i, j=1,2, \ldots, N \\
A_{i j}^{(m)}=-\sum_{j=1, j \neq i}^{N} A_{i j}^{(m)} & \text { For } i=1,2, \ldots, N
\end{array}
$$

In which the following relationship exists:

$$
A_{i j}^{(1)}=\frac{M^{(1)}\left(Z_{i}\right)}{\left(z_{i}-x_{j}\right) M^{(1)}\left(Z_{j}\right)} \quad \text { For } i \neq j
$$

where $M^{(1)}$ is denoted by the following expression: 


$$
M^{(1)}\left(Z_{i}\right)=\prod_{j=1, j \neq i}^{N}\left(z_{i}-z_{j}\right) \quad \text { For } i=1,2, \ldots, N
$$

In the application of the mixed method for plate problems, we need to approximate the first and second order derivatives of functions in the $\mathrm{z}$ direction. To attain better accuracy, the positions of points along the thickness direction are determined according to the Chebyshev-Gauss-Lobatto quadrature mesh size which is proposed by Bert and Malik (1996). That is,

$$
z_{i}=\frac{h}{2}\left\{1-\cos \left[\frac{(i-1) \pi}{\left(N_{z}-1\right)}\right]\right\} \quad \text { For } i=1,2, \ldots, N_{z}
$$

In the next section, the applications of DQ method for 3-D analysis of plates and its combination by FE method have been extensively discussed.

\section{FE-DQ Formulation for Three-Dimensional Elasticity}

\section{1 Equations of Motion}

Consider a thick rectangular isotropic plate with length $\mathrm{a}$, width $\mathrm{b}$ and height $\mathrm{h}$ as shown in Fig. 1, which is subjected to different loadings with various boundary conditions in the Cartesian coordinates. A mixed FE-DQ method has been proposed for the three-dimensional solution of buckling and free vibration of such plates. The solution procedure and the required equations for these cases are discussed in the following sections.

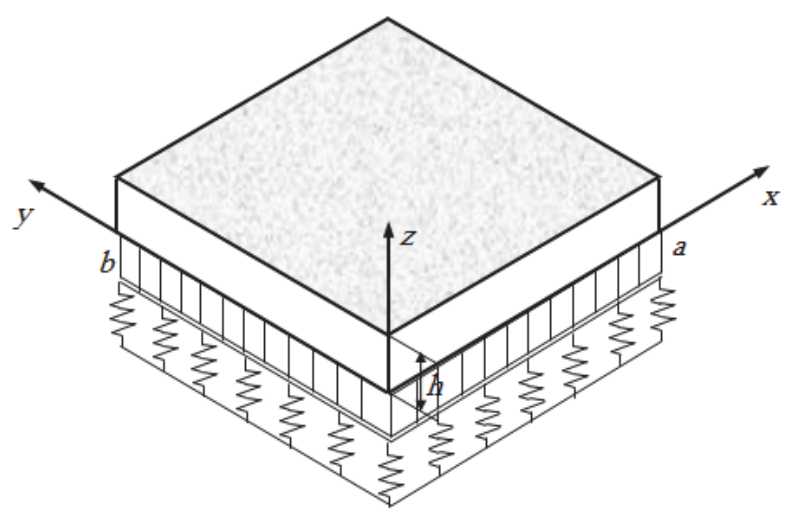

Fig. 1. The geometry of the system under consideration

In order to derive the equations of motion of a plate with assumed boundary conditions for the free vibration and buckling analysis, use is made of the Hamilton's principle, which can be expressed as:

$$
\int_{t_{1}}^{t_{2}}\left(\delta T-\delta U-\delta V_{g}-\delta V_{e f}\right) d t=0
$$

In this article, for 3-D vibration analysis of the plate, both in-plane and out-of-plane inertia of the plate are considered. Therefore, the kinetic energy of the plate becomes:

$$
T=\frac{1}{2} \int_{v} \rho\left(\dot{u}^{2}+\dot{v}^{2}+\dot{w}^{2}\right) d v
$$

where the over dot indicates partial differentiation with respect to time. The strain energy of the plate and the potential energy of elastic foundation are 


$$
\begin{aligned}
U & =\frac{1}{2} \iiint_{v} \sigma_{i j} \varepsilon_{i j} d v, \\
V_{e f} & =\left.\frac{1}{2} \int_{0}^{a} \int_{0}^{b}\left[k_{1} w^{2}+k_{2}\left(\left(\frac{\partial w}{\partial x}\right)^{2}+\left(\frac{\partial w}{\partial y}\right)^{2}\right)\right]\right|_{z=0} d x d y,
\end{aligned}
$$

where $k_{1}$ and $k_{2}$ are Winkler and shearing layer elastic coefficients of foundation respectively. The potential energy of the applied in-plane stresses is

$$
V_{g}=\frac{1}{2} \iiint_{v}\left\{P_{x}\left[\left(\frac{\partial u}{\partial x}\right)^{2}+\left(\frac{\partial w}{\partial x}\right)^{2}+\left(\frac{\partial v}{\partial x}\right)^{2}\right]+P_{y}\left[\left(\frac{\partial u}{\partial y}\right)^{2}+\left(\frac{\partial w}{\partial y}\right)^{2}+\left(\frac{\partial v}{\partial y}\right)^{2}\right]\right\} d v \text {. }
$$

As shown in Fig. 2, $P_{x}$ an $P_{y}$ represent the applied stresses along the x and y-axis, respectively. In this expression the terms in the bracket represent the in-plane and the out-of-plane destabilizing influence of the applied stresses (Dehghan \& Baradaran, 2011), (Ventsel, 2001). Substituting Eqs. (811) into Eq. (7) yields the following integral equation.

$$
\begin{aligned}
& \int_{t_{1}}^{t_{2}}\left\{\int _ { 0 } ^ { h } \int _ { 0 } ^ { a } \int _ { 0 } ^ { b } \left[\rho(\dot{u} \delta \dot{u}+\dot{v} \delta \dot{v}+\dot{w} \delta \dot{w})-\left(\sigma_{x x} \delta \varepsilon_{x x}+\sigma_{y y} \delta \varepsilon_{y y}+\sigma_{z z} \delta \varepsilon_{z z}+\sigma_{y z} \delta \gamma_{y z}+\sigma_{x z} \delta \gamma_{x z}+\sigma_{x y} \delta \gamma_{x y}\right)\right.\right. \\
& \left.-p_{x}\left(\frac{\partial u}{\partial x} \frac{\partial \delta u}{\partial x}+\frac{\partial v}{\partial x} \frac{\partial \delta v}{\partial x}+\frac{\partial w}{\partial x} \frac{\partial \delta w}{\partial x}\right)-p_{y}\left(\frac{\partial u}{\partial y} \frac{\partial \delta u}{\partial y}+\frac{\partial v}{\partial y} \frac{\partial \delta v}{\partial y}+\frac{\partial w}{\partial y} \frac{\partial \delta w}{\partial y}\right)\right] d x d y d z \\
& \left.-\left.\int_{0}^{a} \int_{0}^{b}\left[k_{1} w \delta w+k_{2}\left(\frac{\partial w}{\partial x} \frac{\partial \delta w}{\partial x}+\frac{\partial w}{\partial y} \frac{\partial \delta w}{\partial y}\right)\right]\right|_{z=0} d x d y\right\} d t=0
\end{aligned}
$$

The constitutive relations for an isotropic body in three-dimensional elasticity can be written as,

$$
\left\{\begin{array}{l}
\sigma_{x x} \\
\sigma_{y y} \\
\sigma_{z z} \\
\sigma_{y z} \\
\sigma_{z x} \\
\sigma_{x y}
\end{array}\right\}=\left[\begin{array}{cccccc}
C_{11} & C_{12} & C_{12} & 0 & 0 & 0 \\
C_{12} & C_{11} & C_{12} & 0 & 0 & 0 \\
C_{12} & C_{12} & C_{11} & 0 & 0 & 0 \\
0 & 0 & 0 & C_{44} & 0 & 0 \\
0 & 0 & 0 & 0 & C_{55} & 0 \\
0 & 0 & 0 & 0 & 0 & C_{66}
\end{array}\right]\left\{\begin{array}{c}
\varepsilon_{x x} \\
\varepsilon_{y y} \\
\varepsilon_{z z} \\
\gamma_{y z} \\
\gamma_{z x} \\
\gamma_{x y}
\end{array}\right\},
$$

where

$$
\left[\begin{array}{llllll}
\varepsilon_{x x} & \varepsilon_{y y} & \varepsilon_{z z} & \gamma_{y z} & \gamma_{z x} & \gamma_{x y}
\end{array}\right]=\left[\begin{array}{lll}
\frac{\partial u}{\partial x} & \frac{\partial v}{\partial y} & \frac{\partial w}{\partial z}
\end{array}\left(\frac{\partial v}{\partial z}+\frac{\partial w}{\partial y}\right)\left(\frac{\partial u}{\partial z}+\frac{\partial w}{\partial x}\right)\left(\frac{\partial u}{\partial y}+\frac{\partial v}{\partial x}\right)\right] .
$$

The elastic constant coefficients $C_{i j}$, in terms of Young's modulus and Poisson's ratio are given as,

$$
C_{44}=C_{55}=C_{66}=\mu=G=\frac{E}{2(1+v)}, C_{12}=\lambda, C_{11}=\lambda+2 \mu
$$


where $\lambda=\frac{E v}{(1-2 v)(1+v)}, \quad \mu=\frac{E}{2(1+v)}$.

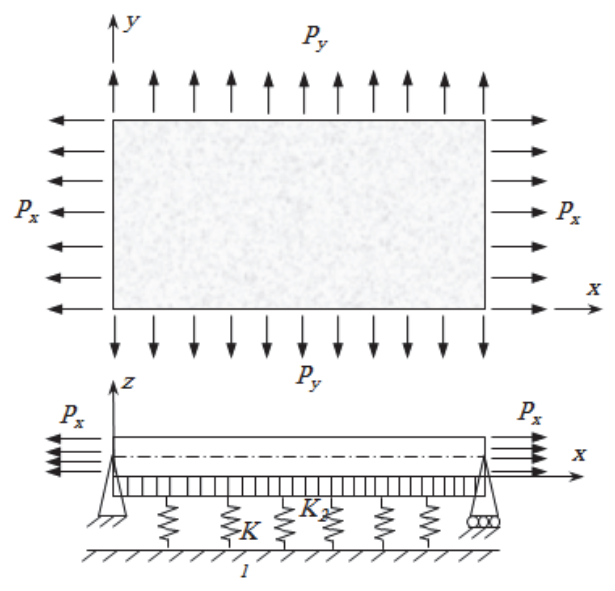

Fig. 2. Geometry and loading condition of plate

As shown in Fig. 3 the problem domain in the $x-y$ plane is divided into a set of $N e$ rectangular quadratic elements. The variational statement of equations require that in each element both in-plane and transverse displacement be only once differentiable and hence $C^{0}$-continuous. The displacement components are approximated by the following interpolations,

$$
\begin{aligned}
& u(x, y, z, t)=\sum_{j=1}^{N} \phi_{j}(x, y) U_{j}(z, t), \quad v(x, y, z, t)=\sum_{j=1}^{N} \phi_{j}(x, y)_{j}(z, t), \\
& w(x, y, z, t)=\sum_{j=1}^{N} \phi_{j}(x, y) W_{j}(z, t),
\end{aligned}
$$

where $j=1,2, \ldots, N$ and $N$ is referred to as the number of nodes distributed on the $\mathrm{x}-\mathrm{y}$ plane of the plate. The functions $\varphi_{j}(x, y)$ are the global Lagrange interpolation functions. In order to obtain the equations of motion at each node, the approximation functions of Eq. (15) are substituted into Eq. (12) and integrating by part with respect to the $\mathrm{z}$ variable. For node $(i)$ with $i=1,2, \ldots, N$ we have,

$$
\begin{aligned}
& \delta U_{i}: \sum_{j=1}^{N} A_{55}^{i j} \frac{\partial^{2} U_{j}}{\partial z^{2}}+\sum_{j=1}^{N}\left(B_{55}^{i j}-B_{12}^{j i}\right) \frac{\partial W_{j}}{\partial z}-\sum_{j=1}^{N}\left(H_{11}^{i j}+F_{66}^{i j}\right) U_{j}-\sum_{j=1}^{N}\left(E_{12}^{i j}+E_{66}^{j i}\right) V_{j}-P_{x} \sum_{j=1}^{N} D^{i j} U_{j} \\
& -P_{y} \sum_{j=1}^{N} B^{i j} U_{j}=\sum_{j=1}^{N} M_{11}^{i j} \ddot{U}_{j} \\
& \delta V_{i}: \sum_{j=1}^{N} A_{44}^{i j} \frac{\partial^{2} V_{j}}{\partial z^{2}}+\sum_{j=1}^{N}\left(D_{44}^{i j}-D_{12}^{j i}\right) \frac{\partial W_{j}}{\partial z}-\sum_{j=1}^{N}\left(E_{12}^{j i}+E_{66}^{i j}\right) U_{j}-\sum_{j=1}^{N}\left(F_{11}^{i j}+H_{66}^{j i}\right) V_{j}-P_{x} \sum_{j=1}^{N} D^{i j} V_{j} \\
& -P_{y} \sum_{j=1}^{N} B^{i j} V_{j}=\sum_{j=1}^{N} M_{11}^{i j} \ddot{V}_{j} \\
& \delta W_{i}: \sum_{j=1}^{N} A_{11}^{i j} \frac{\partial^{2} W_{j}}{\partial z^{2}}+\sum_{j=1}^{N}\left(B_{12}^{i j}-B_{55}^{j i}\right) \frac{\partial U_{j}}{\partial z}+\sum_{j=1}^{N}\left(D_{12}^{i j}-D_{44}^{j i}\right) \frac{\partial V_{j}}{\partial z}-\sum_{j=1}^{N}\left(F_{44}^{i j}+H_{55}^{i j}\right) W_{j}-P_{x} \sum_{j=1}^{N} D^{i j} W_{j} \\
& -P_{y} \sum_{j=1}^{N} B^{i j} W_{j}=\sum_{j=1}^{N} M_{11}^{i j} \ddot{W}_{j}
\end{aligned}
$$

The related boundary conditions at the lower and upper surface of the plate $(z=0, h)$ for the above equations are also obtained as 
Either $\delta U_{i}=0, \quad$ or $\begin{cases}\sum_{j=1}^{N} A_{55}^{i j} \frac{\partial U_{j}}{\partial z}+\sum_{j=1}^{N} B_{55}^{i j} W_{j}=0 & \text { at } z=0 \\ \sum_{j=1}^{N} A_{55}^{i j} \frac{\partial U_{j}}{\partial z}+\sum_{j=1}^{N} B_{55}^{i j} W_{j}=0 & \text { at } z=h\end{cases}$

Either $\quad \delta V_{i}=0, \quad$ or $\begin{cases}\sum_{j=1}^{N} A_{44}^{i j} \frac{\partial V_{j}}{\partial z}+\sum_{j=1}^{N} D_{44}^{i j} W_{j}=0 & \text { at } z=0 \\ \sum_{j=1}^{N} A_{44}^{i j} \frac{\partial V_{j}}{\partial z}+\sum_{j=1}^{N} D_{44}^{i j} W_{j}=0 & \text { at } z=h\end{cases}$

Either $\delta W_{i}=0$,

$$
\text { or }\left\{\begin{array}{cl}
\sum_{j=1}^{N} A_{11}^{i j} \frac{\partial W_{j}}{\partial z}+\sum_{j=1}^{N} D_{12}^{i j} V_{j}+\sum_{j=1}^{N} B_{12}^{i j} U_{j}-\sum_{j=1}^{N}\left(A_{w}^{i j}+D_{g}^{i j}+B_{g}^{i j}\right) W_{j}=0 & \text { at } z=0 \\
\sum_{j=1}^{N} A_{11}^{i j} \frac{\partial W_{j}}{\partial z}+\sum_{j=1}^{N} D_{12}^{i j} V_{j}+\sum_{j=1}^{N} B_{12}^{i j} U_{j}=0 & \text { at } z=h
\end{array}\right.
$$

where

$$
\begin{array}{cl}
A_{m n}^{i j}=\int_{0}^{a} \int_{0}^{b} c_{m n} \phi_{i} \phi_{j} d y d x, & B_{m n}^{i j}=\int_{0}^{a} \int_{0}^{b} c_{m n} \phi_{i}\left(\frac{\partial \phi_{j}}{\partial x}\right) d y d x, \\
D_{m n}^{i j}=\int_{0}^{a} \int_{0}^{b} c_{m n} \phi_{i}\left(\frac{\partial \phi_{j}}{\partial y}\right) d y d x, & H_{m n}^{i j}=\int_{0}^{a} \int_{0}^{b} c_{m n}\left(\frac{\partial \phi_{i}}{\partial x} \frac{\partial \phi_{j}}{\partial x}\right) d y d x, \\
F_{m n}^{i j}=\int_{0}^{a} \int_{0}^{b} c_{m n}\left(\frac{\partial \phi_{i}}{\partial y} \frac{\partial \phi_{j}}{\partial y}\right) d y d x, & E_{m n}^{i j}=\int_{0}^{a} \int_{0}^{b} c_{m n}\left(\frac{\partial \phi_{i}}{\partial x} \frac{\partial \phi_{j}}{\partial y}\right) d y d x, \\
M_{11}^{i j}=\int_{0}^{a} \int_{0}^{b} \rho \phi_{i} \phi_{j} d y d x & A_{w}^{i j}=\int_{0}^{a} \int_{0}^{b} K_{1} \phi_{i} \phi_{j} d y d x, \\
D_{g}^{i j}=\int_{0}^{a} \int_{0}^{b} K_{2}\left(\frac{\partial \phi_{i}}{\partial x} \frac{\partial \phi_{j}}{\partial x}\right) d y d x, & B_{g}^{i j}=\int_{0}^{a} \int_{0}^{b} K_{2}\left(\frac{\partial \phi_{i}}{\partial y} \frac{\partial \phi_{j}}{\partial y}\right) d y d x,
\end{array}
$$

As mentioned previously, the FE-approximation can be used as an appropriate method to calculate these integrations.

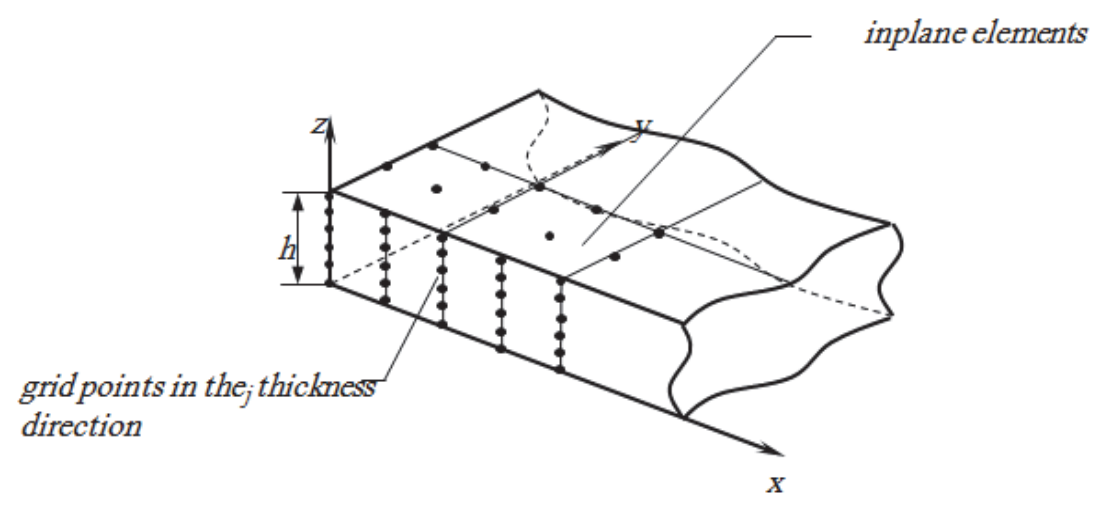

Fig. 3. FE quadratic elements with DQ discretization 


\section{2 DQ Discretization}

At this stage, the DQ method has been used to discretize the resulting system of equations in the thickness direction (along the z-axis). According to the method, at each quadratic element node a set of $N_{z}$ grid points is used to discretize the domain in the thickness direction. By using Eq. (2-6), the resulting system of equations for each node $(k), k=2,3, \ldots, N_{z}-1$ can be expressed as Eq. (16):

$$
\begin{aligned}
& \sum_{j=1}^{N} \sum_{m=1}^{N_{z}} A_{55}^{i j} A_{k m}^{(2)} U_{j m}+\sum_{j=1}^{N} \sum_{m=1}^{N_{z}}\left(B_{55}^{i j}-B_{12}^{j i}\right) A_{k m}^{(1)} W_{j m}-\sum_{j=1}^{N}\left(H_{11}^{i j}+F_{66}^{i j}\right) U_{j k}-\sum_{j=1}^{N}\left(E_{12}^{i j}+E_{66}^{j i}\right) V_{j k} \\
& -P_{x} \sum_{j=1}^{N} D^{i j} U_{j k}-P_{y} \sum_{j=1}^{N} B^{i j} U_{j k}=\sum_{j=1}^{N} M_{11}^{i j} \ddot{U}_{j k}
\end{aligned}
$$

Eq. (17):

$$
\begin{aligned}
& \sum_{j=1}^{N} \sum_{m=1}^{N_{z}} A_{44}^{i j} A_{k m}^{(2)} V_{j m}+\sum_{j=1}^{N} \sum_{m=1}^{N_{z}}\left(D_{44}^{i j}-D_{12}^{j i}\right) A_{k m}^{(1)} W_{j m}-\sum_{j=1}^{N}\left(E_{12}^{j i}+E_{66}^{i j}\right) U_{j k}-\sum_{j=1}^{N}\left(F_{11}^{i j}+H_{66}^{j i}\right) V_{j k} \\
& -P_{x} \sum_{j=1}^{N} D^{i j} V_{j k}-P_{y} \sum_{j=1}^{N} B^{i j} V_{j k}=\sum_{j=1}^{N} M_{11}^{i j} \ddot{V}_{j k}
\end{aligned}
$$

Eq. (18):

$$
\begin{aligned}
& \sum_{j=1}^{N} \sum_{m=1}^{N_{z}} A_{11}^{i j} A_{k m}^{(2)} W_{j m}+\sum_{j=1}^{N} \sum_{m=1}^{N_{z}}\left(B_{12}^{i j}-B_{55}^{j i}\right) A_{k m}^{(1)} U_{j m}+\sum_{j=1}^{N} \sum_{m=1}^{N_{z}}\left(D_{12}^{i j}-D_{44}^{j i}\right) A_{k m}^{(1)} V_{j m}-\sum_{j=1}^{N}\left(F_{44}^{i j}+H_{55}^{i j}\right) W_{j k} \\
& -P_{x} \sum_{j=1}^{N} D^{i j} W_{j k}-P_{y} \sum_{j=1}^{N} B^{i j} W_{j k}=\sum_{j=1}^{N} M_{11}^{i j} \ddot{W}_{j k}
\end{aligned}
$$

In a similar manner, for nodes $(k), k=1, \mathrm{~N}_{z}$, at the upper and lower surfaces of the plate we have the following equations:

$$
\begin{array}{lll}
\text { Either } \quad U_{i k}=0 & \text { or } \begin{cases}\sum_{j=1}^{N} \sum_{m=1}^{N_{z}} A_{55}^{i j} A_{k m}^{(1)} U_{j m}+\sum_{j=1}^{N} B_{55}^{i j} W_{j k}=0 & \text { For } k=1 \\
\sum_{j=1}^{N} \sum_{m=1}^{N_{z}} A_{55}^{i j} A_{k m}^{(1)} U_{j m}+\sum_{j=1}^{N} B_{55}^{i j} W_{j k}=0 & \text { For } k=N_{z}\end{cases} \\
\text { Either } \quad V_{i k}=0 & \text { or } \begin{cases}\sum_{j=1}^{N} \sum_{m=1}^{N_{z}} A_{44}^{i j} A_{k m}^{(1)} V_{j m}+\sum_{j=1}^{N} D_{44}^{i j} W_{j k}=0 & \text { For } k=1 \\
\sum_{j=1}^{N} \sum_{m=1}^{N_{z}} A_{44}^{i j} A_{k m}^{(1)} V_{j m}+\sum_{j=1}^{N} D_{44}^{i j} W_{j k}=0 & \text { For } k=N_{z}\end{cases}
\end{array}
$$

Either $W_{i k}=0$

$$
\text { or } \begin{cases}\sum_{j=1}^{N} \sum_{m=1}^{N_{z}} A_{11}^{i j} A_{k m}^{(1)} W_{j m}+\sum_{j=1}^{N} D_{12}^{i j} V_{j k}+\sum_{j=1}^{N} B_{12}^{i j} U_{j k}-\sum_{j=1}^{N}\left(A_{w}^{i j}+B_{g}^{i j}+D_{g}^{i j}\right) W_{j k}=0 & \text { For } k=1 \\ \sum_{j=1}^{N} \sum_{m=1}^{N_{z}} A_{11}^{i j} A_{k m}^{(1)} W_{j m}+\sum_{j=1}^{N} D_{12}^{i j} V_{j k}+\sum_{j=1}^{N} B_{12}^{i j} U_{j k}=0 & \text { For } k=N_{z}\end{cases}
$$


As shown earlier, the resulting system of equations is divided into the domain-type and the boundary -type equations. In order to provide a unified program for the analysis, the degrees of freedom should also be separated to domain and boundary type degrees of freedom. Therefore, the degrees of freedom in the solution domain can be separately given by:

$$
\begin{aligned}
& \left\{U_{d}\right\}=\left\{\begin{array}{c}
U_{12} \\
U_{13} \\
\vdots \\
U_{N\left(N_{z}-1\right)}
\end{array}\right\}, \quad\left\{V_{d}\right\}=\left\{\begin{array}{c}
V_{12} \\
V_{13} \\
\vdots \\
V_{N\left(N_{z}-1\right)}
\end{array}\right\}, \quad\left\{W_{d}\right\}=\left\{\begin{array}{c}
W_{12} \\
W_{13} \\
\vdots \\
W_{N\left(N_{z}-1\right)}
\end{array}\right\}, \\
& \left\{U_{b}\right\}=\left\{\begin{array}{c}
U_{11} \\
U_{21} \\
\vdots \\
U_{N N_{z}}
\end{array}\right\}, \quad\left\{V_{b}\right\}=\left\{\begin{array}{c}
V_{11} \\
V_{21} \\
\vdots \\
V_{N N_{z}}
\end{array}\right\}, \quad\left\{W_{b}\right\}=\left\{\begin{array}{c}
W_{11} \\
W_{21} \\
\vdots \\
W_{N N_{z}}
\end{array}\right\},
\end{aligned}
$$

where, $W_{m n}=W_{m}\left(z_{n}, t\right), V_{m n}=V_{m}\left(z_{n}, t\right), U_{m n}=U_{m}\left(z_{n}, t\right)$. Based on the aforementioned division for degrees of freedom, the equations of motion and the related boundary conditions can be expressed in the matrix form as:

$$
\begin{aligned}
& {\left[S_{d b}\right]\left\{\begin{array}{l}
\left\{U_{b}\right\} \\
\left\{V_{b}\right\} \\
\left\{W_{b}\right\}
\end{array}\right\}+\left[S_{d d}\right]\left\{\begin{array}{l}
\left\{U_{d}\right\} \\
\left\{V_{d}\right\} \\
\left\{W_{d}\right\}
\end{array}\right\}-P[G]\left\{\begin{array}{l}
\left\{U_{d}\right\} \\
\left\{V_{d}\right\} \\
\left\{W_{d}\right\}
\end{array}\right\}=[M]\left\{\begin{array}{c}
\left\{\ddot{U}_{d}\right\} \\
\left\{\ddot{V}_{d}\right\} \\
\left\{\ddot{W}_{d}\right\}
\end{array}\right\},} \\
& {\left[S_{b b}\right]\left\{\begin{array}{l}
\left\{U_{b}\right\} \\
\left\{V_{b}\right\} \\
\left\{W_{b}\right\}
\end{array}\right\}+\left[S_{b d}\right]\left\{\begin{array}{l}
\left\{U_{d}\right\} \\
\left\{V_{d}\right\} \\
\left\{W_{d}\right\}
\end{array}\right\}=\{0\} .}
\end{aligned}
$$

By eliminating the boundary degrees of freedom from Eq. (29) using Eq. (30), we have

$$
[S]\left\{\begin{array}{l}
\left\{U_{d}\right\} \\
\left\{V_{d}\right\} \\
\left\{W_{d}\right\}
\end{array}\right\}-P[G]\left\{\begin{array}{l}
\left\{U_{d}\right\} \\
\left\{V_{d}\right\} \\
\left\{W_{d}\right\}
\end{array}\right\}=[M]\left\{\begin{array}{c}
\left\{\ddot{U}_{d}\right\} \\
\left\{\ddot{V}_{d}\right\} \\
\left\{\ddot{W}_{d}\right\}
\end{array}\right\},
$$

where

$$
[S]=\left[S_{d d}\right]-\left[S_{d b}\right]\left[S_{b b}\right]^{-1}\left[S_{b d}\right]
$$

The parameter $\mathrm{P}$ in the equations refers to the resultant of buckling loads $P_{x}$ and $P_{y}$ which are considered along the $\mathrm{x}$ - and $\mathrm{y}$-axis, respectively. For the free vibration and buckling analysis, the following harmonic solution should be inserted in the equations:

$$
\left\{\begin{array}{l}
\left\{U_{d}\right\} \\
\left\{V_{d}\right\} \\
\left\{W_{d}\right\}
\end{array}\right\}=\left\{\begin{array}{l}
\left\{\bar{U}_{d}\right\} \\
\left\{\bar{V}_{d}\right\} \\
\left\{\bar{W}_{d}\right\}
\end{array}\right\} e^{j w t}
$$


where ${ }_{\omega}$ denotes the natural frequency of vibration in radians and $j=\sqrt{-1}$. Substituting the harmonic displacements of Eq. (33), Eq. (31) becomes

$$
[S]\left\{\begin{array}{l}
\left\{\bar{U}_{d}\right\} \\
\left\{\overline{V_{d}}\right\} \\
\left\{\bar{W}_{d}\right\}
\end{array}\right\}-P[G]\left\{\begin{array}{l}
\left\{\bar{U}_{d}\right\} \\
\left\{\overline{V_{d}}\right\} \\
\left\{\bar{W}_{d}\right\}
\end{array}\right\}+\omega^{2}[M]\left\{\begin{array}{l}
\left\{\bar{U}_{d}\right\} \\
\left\{\overline{V_{d}}\right\} \\
\left\{\bar{W}_{d}\right\}
\end{array}\right\}=0
$$

The second terms indicates the influence of the applied in-plane loads on the free vibration of the plates. The critical buckling load $P_{c r}$ is regarded as the in-plane load $P$ associated with the zero natural frequency. Consequently, by eliminating the term containing the frequency parameter (the last term), we can arrive at the eigenvalue equation for the buckling analysis as,

$$
[S]\left\{\begin{array}{l}
\left\{\bar{U}_{d}\right\} \\
\left\{\bar{V}_{d}\right\} \\
\left\{\bar{W}_{d}\right\}
\end{array}\right\}-P[G]\left\{\begin{array}{l}
\left\{\bar{U}_{d}\right\} \\
\left\{\bar{V}_{d}\right\} \\
\left\{\bar{W}_{d}\right\}
\end{array}\right\}=0
$$

\section{3 Defining the Boundary Conditions}

The boundary conditions at the top $(z=h)$ and the bottom $(z=0)$ surfaces of the plate are exactly satisfied in Eq. (25-27). To compare the results obtained by the current method with the results in the literature, three sets of boundary conditions, i.e., simply supported (SSSS), clamped (CCCC) and mixed simply supported and clamped (SCSC) are considered at the edges of the plate. For example in SCSC plate the edges $x=0, a$ are simply supported and $y=0, b$ are clamped. These three boundary conditions are precisely defined as follows.

(i) Simply supported (SSSS)

$$
\begin{aligned}
& \text { on } x=0 \text { and } a: \sigma_{\mathrm{x}}=0, v=0, w=0 \\
& \text { on } y=0 \text { and } b: u=0, \sigma_{y}=0, w=0 .
\end{aligned}
$$

(ii) Clamped (CCCC)

$$
\text { on } x=0, a \text { and } y=0, b: u=0, v=0, w=0
$$

(iii) Mixed simply supported and clamped (SCSC)

$$
\begin{aligned}
& \text { on } x=0 \text { and } a: \sigma_{\mathrm{x}}=0, v=0, w=0 \\
& \text { on } x=0, a \text { and } y=0, b: u=0, v=0, w=0
\end{aligned}
$$

\section{Numerical Results and Discussion}

In this section, several numerical examples have been presented to illustrate the efficiency and accuracy of the proposed method in 3-D analysis of thick rectangular plates with various boundary conditions. Numerical examples are presented in two parts in the following subsections. The numerical examples in the first section are those for which solution results with other solution methods are available in the literature; therefore, the comparisons of the results reveal the validity and accuracy of 
the proposed FE-DQ method. In the second part, some numerical examples including uniaxial and biaxial buckling, and free vibration analysis are carried out through FE-DQ method and the results of these numerical examples are presented. In an attempt to present the results in a better way, four nondimensional parameters are defined:

$$
\bar{\omega}=\frac{\omega b^{2}}{\pi^{2}} \sqrt{\frac{p h}{D}}, \Lambda=P_{X} \frac{b^{2} h}{\pi^{2} D}, \bar{K}_{1}=\frac{K_{1} a^{4}}{D}, \bar{K}_{2}=\frac{K_{2} a^{2}}{D}
$$

where, $\bar{a}$ and $\Lambda$ are non-dimensional frequency and non-dimensional buckling load in the $\mathrm{x}$ direction. $\bar{K}_{1}$ and $\bar{K}_{2}$ are the non-dimensional elastic coefficients of Winkler and Pasternak foundations, respectively. $D$ is the bending stiffness of the plate which is defined as $D=E h^{3} /\left(12\left(1-v^{2}\right)\right)$. It should be noted that in the grid-size notation used to represent the degree of discretization in FE-DQ method (for example $7 \times 7 \times 7$ ), the first two digits refer to the number of elements along the $\mathrm{x}$ - and $\mathrm{y}$-axis, respectively, and the third digit specifies the DQ grid-size along the z-axis. The poisson's ratio is taken as 0.3 .

\section{1 Comparison of the Results with Other Solution Methods}

Using Ref. (Matsunaga, 2000) as the exact solution, effects of mesh density on the first three natural frequencies of square SSSS plates supported by elastic foundation are investigated in Fig. 4. The different mixed numbers of $2 \times 2 \ldots 9 \times 9$ in-plane elements are considered in the solution domain. The error percentage is calculated using the following formula

$$
\% \text { Error }=\left|\frac{\text { present solution }- \text { exact solution }}{\text { exact solution }}\right| \times 100
$$

Fig. 5 returns the total CPU time (in seconds) used in different mesh-sizes of in-plane domain for the previous example. It should be noted that, the entire analysis is programmed to run on Core ${ }^{\mathrm{TM}}$ processor using MATLAB software.

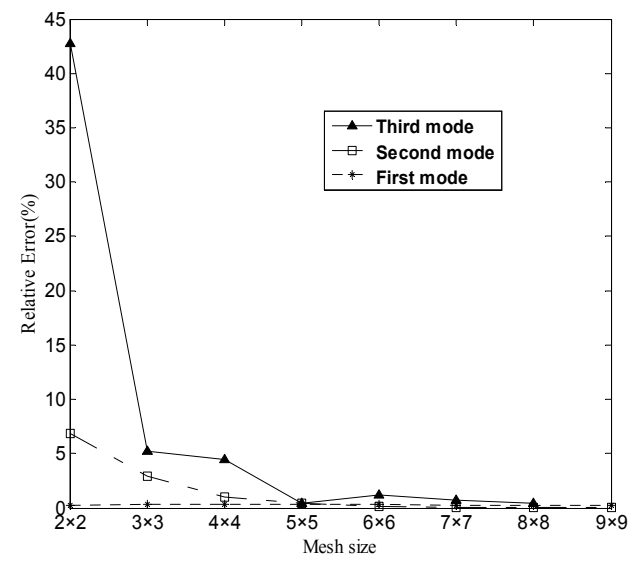

Fig. 4. The percent of relative error for the first three transverse non-dimensional natural frequencies of SSSS plate $\left(N_{z}=7, \overline{k_{1}}=0, \overline{k_{2}}=10\right.$, $h / b=0.2)$

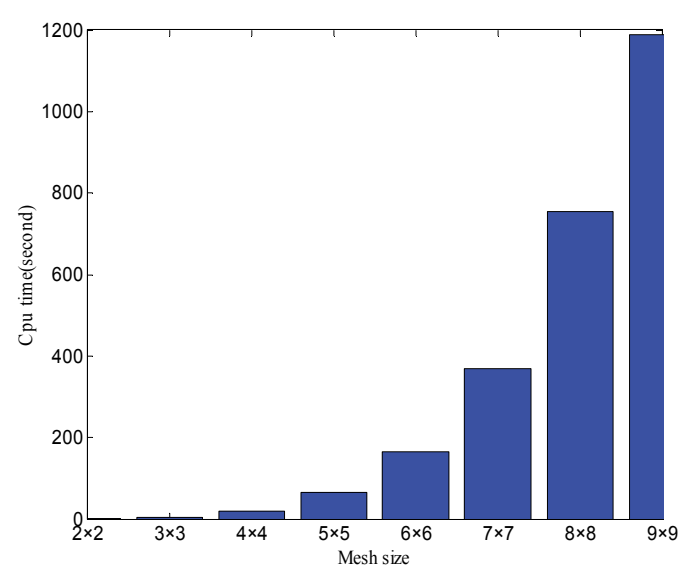

Fig. 5. The total CPU time (in seconds) at different mesh-sizes

Three-dimensional uniaxial-buckling behaviors of thick square plates with thicknesses ratios of 0.2 and 0.5 resting on two parameter elastic foundations are investigated in Table 1 . The results are represented for different elastic coefficients of Winkler and Pasternak. Several preliminary test examples with the FE-DQ method showed that good accuracy can be obtained with the mesh size of $9 \times 9 \times 7$. Therefore, the $9 \times 9 \times 7$ mesh-size is selected as a convenient mesh in this table. In Table 1 , the 
critical buckling loads given by FE-DQ method are compared with higher-order and Mindlin plate theories. It can be observed from the table that for thickness ratio of 0.2, the higher-order and Mindlin theories of the plate are exactly followed by results obtained from the FE-DQ method. However, in thickness ratio of 0.5 as expected the results follow only the higher-order theory of plate. Furthermore, it can be concluded from the table that for the thickness ratio of 0.5 and higher elastic coefficients the critical buckling loads remain unchanged. In Table 2 the non-dimensional natural frequencies of SSSS thin and moderately thick plates resting on Winkler elastic foundation $\left(\bar{k}_{2}=0\right)$ are presented. In order to investigate the convergence behavior of the FE-DQ method, three different grid-sizes of $5 \times 5 \times 5$, $7 \times 7 \times 7$ and $9 \times 9 \times 9$, are employed and the results are compared with the analytical solutions. It is shown that the method has an acceptable accuracy and convergence. As a simpler way of comparison, the eigenfrequency parameter $\bar{\omega}_{m, n}(m, n=1,2,3, \ldots)$ as defined in Eq. (39) is used to denote the flexural modes of a plate, where subscripts $m$ and $n$ mean the wave numbers in the $x$ and $y$ directions, respectively.

Table 1. Critical buckling factors, $\Lambda_{c r}=P_{x} b^{2} h /\left(\pi^{2} D\right)$, of the uniaxially compressed square SSSS plates. $(\nu=0.3)\left(N_{x}=9, N_{y}=9, N_{z}=7\right)$

\begin{tabular}{|c|c|c|c|c|c|}
\hline \multirow[b]{2}{*}{$\frac{h}{b}$} & \multirow[b]{2}{*}{$\overline{k_{1}}$} & \multirow[b]{2}{*}{$\bar{k}_{2}$} & \multicolumn{3}{|l|}{$\Lambda_{c r}$} \\
\hline & & & $\begin{array}{l}\text { Mindlin } \\
\text { (Matsunaga, 2000) }\end{array}$ & $\begin{array}{l}\text { Higher-order } \\
\text { (Matsunaga, 2000) }\end{array}$ & $\begin{array}{l}\text { Present } \\
\text { method }\end{array}$ \\
\hline \multirow[t]{12}{*}{0.2} & 0 & 0 & 3.1255 & 3.1533 & 3.1392 \\
\hline & 10 & & 3.2238 & 3.2471 & 3.2326 \\
\hline & $10^{2}$ & & 3.9751 & 4.0180 & 3.9940 \\
\hline & $10^{3}$ & & 5.8906 & 5.8408 & 5.8031 \\
\hline & $10^{4}$ & & 8.8656 & 8.6580 & 8.6263 \\
\hline & $10^{5}$ & & 8.8656 & 9.1642 & 9.2894 \\
\hline & 0 & 10 & 4.9160 & 4.8523 & 4.8270 \\
\hline & 10 & & 4.9399 & 4.8731 & 4.8487 \\
\hline & $10^{2}$ & & 5.1549 & 5.0594 & 5.0361 \\
\hline & $10^{3}$ & & 6.9236 & 6.6206 & 6.5919 \\
\hline & $10^{4}$ & & 9.8788 & 8.9134 & 8.8777 \\
\hline & $10^{5}$ & & 9.8788 & 9.1644 & 9.2950 \\
\hline \multirow[t]{12}{*}{0.5} & 0 & 0 & 1.3027 & 1.3586 & 1.3514 \\
\hline & 10 & & 1.3267 & 1.3782 & 1.3702 \\
\hline & $10^{2}$ & & 1.4125 & 1.4702 & 1.4527 \\
\hline & $10^{3}$ & & 1.4185 & 1.4371 & 1.4661 \\
\hline & $10^{4}$ & & 1.4185 & 1.4732 & 1.4667 \\
\hline & $10^{5}$ & & 1.4185 & 1.4732 & 1.4667 \\
\hline & 0 & 10 & 1.7022 & 1.4732 & 1.4667 \\
\hline & 10 & & 1.7022 & 1.4732 & 1.4667 \\
\hline & $10^{2}$ & & 1.7022 & 1.4732 & 1.4667 \\
\hline & $10^{3}$ & & 1.7022 & 1.4732 & 1.4667 \\
\hline & $10^{4}$ & & 1.7022 & 1.4732 & 1.4667 \\
\hline & $10^{5}$ & & 1.7022 & 1.4732 & 1.4667 \\
\hline
\end{tabular}


Table 2. Comparison of the non-dimensional natural frequency parameters of simply supported thin and moderately plates on Winkler foundation. $\left(\overline{k_{2}}\right)$

\begin{tabular}{|c|c|c|c|c|c|}
\hline$\frac{h}{b}$ & $\overline{k_{1}}$ & Grid size/elements & $\bar{\omega}_{1}$ & $\bar{\omega}_{12}$ & $\bar{\omega}_{2}$ \\
\hline \multirow[t]{10}{*}{0.01} & $10^{2}$ & $5 \times 5 \times 5$ & 2.2520 & 5.3164 & 8.3492 \\
\hline & & $7 \times 7 \times 7$ & 2.2480 & 5.2136 & 8.1971 \\
\hline & & $9 \times 9 \times 9$ & 2.2450 & 5.1643 & 8.1338 \\
\hline & & (Leissa, 1973) & 2.2420 & 5.1016 & 8.0639 \\
\hline & & (Xiang et al., 1994) & 2.2413 & 5.0971 & 8.0523 \\
\hline & $5 \times 10^{2}$ & $5 \times 5 \times 5$ & 3.0293 & 5.6997 & 8.5913 \\
\hline & & $7 \times 7 \times 7$ & 3.0264 & 5.5933 & 8.4436 \\
\hline & & $9 \times 9 \times 9$ & 3.0242 & 5.5474 & 8.3821 \\
\hline & & (Leissa, 1973) & 3.0221 & 5.4894 & 8.3146 \\
\hline & & (Xiang et al., 1994) & 3.0215 & 5.4850 & 8.3032 \\
\hline \multirow[t]{8}{*}{0.1} & $2 \times 10^{2}$ & $5 \times 5 \times 5$ & 2.3883 & 4.8291 & 7.2458 \\
\hline & & $7 \times 7 \times 7$ & 2.3901 & 4.8139 & 7.2258 \\
\hline & & $9 \times 9 \times 9$ & 2.3903 & 4.8098 & 7.2186 \\
\hline & & (Xiang et al., 1994) & 2.3989 & 4.8194 & 7.2093 \\
\hline & $10^{3}$ & $5 \times 5 \times 5$ & 3.6965 & 5.5690 & 7.7449 \\
\hline & & $7 \times 7 \times 7$ & 3.6976 & 5.5556 & 7.7260 \\
\hline & & $9 \times 9 \times 9$ & 3.6978 & 5.5521 & 7.7193 \\
\hline & & (Xiang et al., 1994) & 3.7212 & 5.5844 & 7.7353 \\
\hline
\end{tabular}

The first three natural frequency parameters for the flexural modes of thin and moderately thick SSSS square plates resting on Pasternak foundation $\left(\bar{k}_{2}=10\right)$ are compared in Table 3 . As mentioned previously, there is no additional assumption in the three-dimensional theory; consequently, it provides more realistic results. Furthermore, it should be considered that the numerical simulation of such a 3$\mathrm{D}$ model has an expensive computational effort. So, the grid-size cannot be fine enough to achieve the desired precision and some differences are appeared between the obtained results and those of exact solution. Computational results for the first three out-of-plane natural frequency parameters of simply supported thick plates on Pasternak foundation $\left(\bar{k}_{2}=10\right)$ are listed in Table 4. As shown, the results obtained for thickness to width ratio 0.5 (thick plate) can be appropriately followed by the proposed method.

Table 3. Comparison of the non-dimensional natural frequency parameters of simply supported thin and moderately thick plates on Pasternak foundation. $\left(\bar{k}_{2}=10\right),(9 \times 9 \times 7)$

\begin{tabular}{llllll}
\hline$\frac{h}{b}$ & $\bar{k}_{1}$ & Method & $\bar{\omega}_{11}$ & $\bar{\omega}_{12}$ & $\bar{\omega}_{2}$ \\
\hline 0.01 & $10^{2}$ & present & 2.6578 & 5.6265 & 8.6152 \\
& & (Xiang et al., 1994) & 2.6551 & 5.5718 & 8.5405 \\
& $5 \times 10^{2}$ & present & 3.3420 & 5.9800 & 8.8500 \\
& & (Xiang et al., 1994) & 3.3400 & 5.9287 & 8.7775 \\
& & & & \\
0.1 & $2 \times 10^{2}$ & present & 2.7721 & 5.2800 & 7.7132 \\
& & (Xiang et al., 1994) & 2.7842 & 5.3043 & 7.7287 \\
& $10^{3}$ & present & 3.9542 & 5.9623 & 8.1816 \\
& & (Xiang et al., 1994) & 3.9805 & 6.0078 & 8.2214 \\
\hline
\end{tabular}


Table 4. Comparison of the first three out-of-plane non-dimensional natural frequency parameters of simply supported thick plates on Pasternak foundation. $\left(\bar{k}_{2}=10\right),(9 \times 9 \times 7)$

\begin{tabular}{|c|c|c|c|c|c|}
\hline$\frac{h}{b}$ & $\bar{k}_{1}$ & Method & $\bar{\omega}_{11}$ & $\bar{\omega}_{12}$ & $\bar{\omega}_{13}$ \\
\hline \multirow[t]{18}{*}{0.2} & 0 & present & 2.2275 & 4.4042 & 7.2759 \\
\hline & & (Xiang et al., 1994) & 2.2505 & 4.4344 & 7.2727 \\
\hline & & (Matsunaga, 2000) & 2.2334 & 4.4056 & 7.2436 \\
\hline & 10 & present & 2.2481 & 4.3967 & 7.2649 \\
\hline & & (Xiang et al., 1994) & 2.2722 & 4.4452 & 7.2792 \\
\hline & & (Matsunaga, 2000) & 2.2539 & 4.4150 & 7.2488 \\
\hline & $10^{2}$ & present & 2.4247 & 4.4973 & 7.3161 \\
\hline & & (Xiang et al., 1994) & 2.4591 & 4.5409 & 7.3373 \\
\hline & & (Matsunaga, 2000) & 2.4300 & 4.4986 & 7.2948 \\
\hline & $10^{3}$ & present & 3.7080 & 5.2276 & 7.7544 \\
\hline & & (Xiang et al., 1994) & 3.8567 & 5.4043 & 7.8938 \\
\hline & & (Matsunaga, 2000) & 3.7112 & 5.2285 & 7.7191 \\
\hline & $10^{4}$ & present & 4.6065 & 7.2759 & 10.0146 \\
\hline & & (Xiang et al., 1994) & 10.076 & 10.644 & 12.067 \\
\hline & & (Matsunaga, 2000) & 4.6127 & 7.2934 & 10.033 \\
\hline & $10^{5}$ & present & 4.6065 & 7.2760 & 10.3053 \\
\hline & & (Xiang et al., 1994) & 17.991 & 20.092 & 21.955 \\
\hline & & (Matsunaga, 2000) & 4.6127 & 7.2934 & 10.314 \\
\hline \multirow[t]{12}{*}{0.5} & 0 & present & 1.5913 & 2.6565 & 3.8241 \\
\hline & & (Matsunaga, 2000) & 1.6462 & 2.6851 & 3.8268 \\
\hline & 10 & present & 1.6055 & 2.6602 & 3.8249 \\
\hline & & (Matsunaga, 2000) & 1.6577 & 2.6879 & 3.8274 \\
\hline & $10^{2}$ & present & 1.7086 & 2.6888 & 3.8316 \\
\hline & & (Matsunaga, 2000) & 1.7437 & 2.7096 & 3.8321 \\
\hline & $10^{3}$ & present & 1.8426 & 2.8000 & 3.8638 \\
\hline & & (Matsunaga, 2000) & 1.8451 & 2.8033 & 3.8578 \\
\hline & $10^{4}$ & present & 1.8426 & 2.8724 & 3.8874 \\
\hline & & (Matsunaga, 2000) & 1.8451 & 2.8739 & 3.8866 \\
\hline & $10^{5}$ & present & 1.8426 & 2.8846 & 3.8902 \\
\hline & & (Matsunaga, 2000) & 1.8451 & 2.8857 & 3.8927 \\
\hline
\end{tabular}

\subsection{Other Numerical Examples}

The natural frequencies of plates partially supported on elastic foundation are investigated by the FE-DQ method in Tables 6 to 8 . In these tables, $l_{x}$ and $l_{y}$ are respectively the length and width of the part of the plate supported by the elastic foundation (Fig. 6).

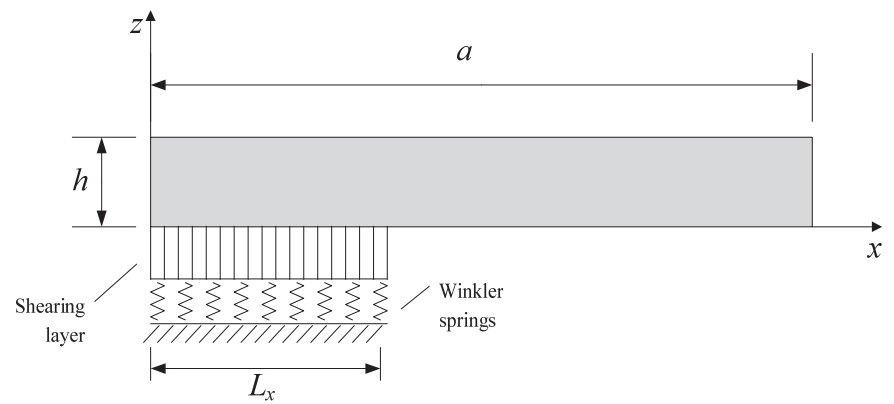

Fig. 6. Partially supported plate on two parameter elastic foundations. 
The effects of elastic foundation area $\left(l_{x} \times l_{y}\right)$ on fundamental frequencies of thick SSSS plates partially supported on two-parameter elastic foundation are listed in Table 5. The frequencies are calculated for different thickness ratios. As the elastic foundation area increase, the frequencies are found to be increasing slightly. Table 6 and Table 7 are also tabulated for fundamental frequencies of SCSC and CCCC plates, respectively. As expected, the CCCC plate has the highest-frequency parameter $\bar{\omega}$, followed by the SCSC and SSSS plates.

Table 5. The first three non-dimensional transverse natural frequencies of partially supported on elastic foundation SSSS plates. $(9 \times 9 \times 7)$

\begin{tabular}{|c|c|c|c|c|c|c|c|c|}
\hline \multirow{2}{*}{$\frac{l_{x}}{a}$} & \multirow{2}{*}{$\frac{l_{y}}{b}$} & \multirow{2}{*}{$\frac{h}{b}$} & \multicolumn{3}{|c|}{$\overline{k_{1}}=10, \overline{k_{2}}=0$} & \multicolumn{3}{|c|}{$\bar{k}_{1}=10, \bar{k}_{2}=10$} \\
\hline & & & $\overline{\bar{\omega}_{11}}$ & $\overline{\bar{\omega}_{12}}$ & $\overline{\bar{\omega}_{22}}$ & $\overline{\bar{\omega}_{11}}$ & $\overline{\bar{\omega}_{12}}$ & $\overline{\bar{\omega}_{22}}$ \\
\hline $1 / 3$ & $1 / 3$ & 0.2 & 1.7708 & 3.8989 & 5.6382 & 1.8147 & 3.9639 & 5.6936 \\
\hline & & 0.3 & 1.5854 & 3.2404 & 4.5018 & 1.6264 & 3.3052 & 4.5557 \\
\hline $1 / 3$ & $2 / 3$ & 0.2 & 1.7740 & 3.8993 & 5.6388 & 1.8849 & 4.0186 & 5.7608 \\
\hline & & 0.3 & 1.5888 & 3.2409 & 4.5025 & 1.6925 & 3.3568 & 4.6233 \\
\hline $2 / 3$ & $2 / 3$ & 0.2 & 1.7869 & 3.9034 & 5.6397 & 1.9797 & 4.1926 & 5.8755 \\
\hline & & 0.3 & 1.6023 & 3.2454 & 4.5035 & 1.7863 & 3.5249 & 4.7337 \\
\hline
\end{tabular}

Table 6. The first three non-dimensional transverse natural frequencies of partially supported on elastic foundation SCSC plates. $(9 \times 9 \times 7)$

\begin{tabular}{|c|c|c|c|c|c|c|c|c|}
\hline \multirow{2}{*}{$\frac{l_{x}}{a}$} & \multirow{2}{*}{$\frac{l_{y}}{b}$} & \multirow{2}{*}{$\frac{h}{b}$} & \multicolumn{3}{|c|}{$\bar{k}_{1}=10, \bar{k}_{2}=0$} & \multicolumn{3}{|c|}{$\bar{k}_{1}=10, \bar{k}_{2}=10$} \\
\hline & & & $\bar{\omega}_{11}$ & $\overline{\bar{\omega}_{12}}$ & $\bar{\omega}_{22}$ & $\overline{\bar{\omega}_{11}}$ & $\bar{\omega}_{12}$ & $\bar{\omega}_{22}$ \\
\hline \multirow{2}{*}{$1 / 3$} & $1 / 3$ & 0.2 & 2.2910 & 4.0689 & 6.0186 & 2.3227 & 4.1037 & 6.0676 \\
\hline & & 0.3 & 1.9023 & 3.3168 & 4.6555 & 1.9354 & 3.3497 & 4.7068 \\
\hline \multirow[t]{2}{*}{$1 / 3$} & $2 / 3$ & 0.2 & 2.2937 & 4.0718 & 6.0192 & 2.3855 & 4.1765 & 6.1110 \\
\hline & & 0.3 & 1.9053 & 3.3200 & 4.6563 & 1.9969 & 3.4204 & 4.7775 \\
\hline \multirow[t]{2}{*}{$2 / 3$} & $2 / 3$ & 0.2 & 2.3042 & 4.0735 & 6.0201 & 2.4687 & 4.3363 & 6.1120 \\
\hline & & 0.3 & 1.9172 & 3.3220 & 4.6573 & 2.0820 & 3.5784 & 4.8873 \\
\hline
\end{tabular}

Table 7. The first three non-dimensional transverse natural frequencies of partially supported on elastic foundation CCCC plates. $(9 \times 9 \times 7)$

\begin{tabular}{|c|c|c|c|c|c|c|c|c|}
\hline \multirow{2}{*}{$\frac{l_{x}}{a}$} & \multirow{2}{*}{$\frac{l_{y}}{b}$} & \multirow{2}{*}{$\frac{h}{b}$} & \multicolumn{3}{|c|}{$\overline{k_{1}}=10, \overline{k_{2}}=0$} & \multicolumn{3}{|c|}{$\bar{k}_{1}=10, \bar{k}_{2}=10$} \\
\hline & & & $\bar{\omega}_{11}$ & $\bar{\omega}_{12}$ & $\bar{\omega}_{22}$ & $\bar{\omega}_{11}$ & $\bar{\omega}_{12}$ & $\bar{\omega}_{22}$ \\
\hline \multirow[t]{2}{*}{$1 / 3$} & $1 / 3$ & 0.2 & 2.7210 & 4.7757 & 6.4039 & 2.7459 & 4.8187 & 6.4484 \\
\hline & & 0.3 & 2.2077 & 3.6653 & 4.8315 & 2.2354 & 3.7180 & 4.9496 \\
\hline \multirow[t]{2}{*}{$1 / 3$} & $2 / 3$ & 0.2 & 2.7230 & 4.7761 & 6.4045 & 2.7999 & 4.8639 & 6.5153 \\
\hline & & 0.3 & 2.2102 & 3.6657 & 4.8323 & 2.2895 & 3.7650 & 4.9504 \\
\hline \multirow[t]{2}{*}{$2 / 3$} & $2 / 3$ & 0.2 & 2.7329 & 4.7799 & 6.4055 & 2.8860 & 5.0406 & 6.6376 \\
\hline & & 0.3 & 2.2216 & 3.6703 & 4.8334 & 2.3765 & 3.9346 & 5.0658 \\
\hline
\end{tabular}

Table 8. Critical buckling factors, $\Lambda=P_{X} b^{2} h / \pi^{2} D$ of the uniaxially square plates on partially supported elastic foundation. $(9 \times 9 \times 7)$

\begin{tabular}{|c|c|c|c|c|c|c|}
\hline \multirow{2}{*}{ Boundary condition } & \multirow{2}{*}{$\frac{l_{x}}{a}$} & \multirow{2}{*}{$\frac{l_{y}}{b}$} & \multicolumn{2}{|c|}{$\overline{k_{1}}=10, \overline{k_{2}}=0$} & \multicolumn{2}{|c|}{$\overline{k_{1}}=10, \bar{k}_{2}=10$} \\
\hline & & & $h / b=0.2$ & 0.3 & 0.2 & 0.3 \\
\hline \multirow[t]{2}{*}{ SSSS } & $1 / 3$ & $1 / 3$ & 3.1430 & 2.5157 & 3.2783 & 2.5660 \\
\hline & $2 / 3$ & $2 / 3$ & 3.1994 & 2.5634 & 3.7904 & 2.8397 \\
\hline \multirow[t]{2}{*}{ SCSC } & $1 / 3$ & $1 / 3$ & 4.1491 & 2.7585 & 4.2059 & 2.8013 \\
\hline & $2 / 3$ & $2 / 3$ & 4.1578 & 2.7665 & 4.5971 & 3.0338 \\
\hline \multirow[t]{2}{*}{$\mathrm{CCCC}$} & $1 / 3$ & $1 / 3$ & 5.0995 & 3.1316 & 5.1256 & 3.1615 \\
\hline & $2 / 3$ & $2 / 3$ & 5.1079 & 3.1394 & 5.5996 & 3.4441 \\
\hline
\end{tabular}


The critical buckling loads of uniaxially and biaxially compressed square plates with different thicknesses and boundary conditions partially supported on elastic foundation are presented in Tables 8 and 9. It can be clearly concluded that an increase in the value of the elastic foundation area always increases the critical buckling load ${ }_{\Lambda}$. The CCCC plate has the highest-buckling load, followed by the SCSC and SSSS plates.

Table 9. Critical buckling factors, of the biaxially square plates on partially supported elastic foundation. $(9 \times 9 \times 7)$

\begin{tabular}{|c|c|c|c|c|c|c|}
\hline \multirow{2}{*}{ Boundary condition } & \multirow{2}{*}{$\frac{l_{x}}{a}$} & \multirow{2}{*}{$\frac{l_{y}}{b}$} & \multicolumn{2}{|c|}{$\overline{k_{1}}=10, \overline{k_{2}}=0$} & \multicolumn{2}{|c|}{$\bar{k}_{1}=10, \bar{k}_{2}=10$} \\
\hline & & & $h / b=0.2$ & 0.3 & 0.2 & 0.3 \\
\hline \multirow[t]{2}{*}{ SSSS } & $1 / 3$ & $1 / 3$ & 1.5690 & 1.2584 & 1.6441 & 1.3185 \\
\hline & $2 / 3$ & $2 / 3$ & 1.5976 & 1.2851 & 1.9433 & 1.5727 \\
\hline \multirow[t]{2}{*}{ SCSC } & $1 / 3$ & $1 / 3$ & 2.4267 & 1.7156 & 2.4849 & 1.7625 \\
\hline & $2 / 3$ & $2 / 3$ & 2.4544 & 1.7422 & 2.8027 & 2.0234 \\
\hline \multirow[t]{2}{*}{$\mathrm{CCCC}$} & $1 / 3$ & $1 / 3$ & 3.0922 & 2.1089 & 3.1357 & 2.1413 \\
\hline & $2 / 3$ & $2 / 3$ & 3.1183 & 2.1340 & 3.5019 & 2.4352 \\
\hline
\end{tabular}

\section{Conclusion}

A mixture of two conventional finite element and differential quadrature methods is used for threedimensional, buckling and free vibration analyses of plates partially resting on elastic foundations. The elastic foundation is described by the Pasternak (two-parameter) model. Mounted columns and single footings that partially occupy the rectangular plates can be considered as an applied example of problem studied here. The governing equations were obtained explicitly via the energy principle. Discretization of the problem at the in-plane (x-y plane) is made by using 2-D FEM through the weak formulation of the equations. DQ approximations have been implemented onto the strong form of the equations along the thickness to discretize the problem in the $\mathrm{z}$ direction. It should be noted that this mixed method deals with the three-dimensional theory of elasticity without any additional assumption about the kinematics of deformation of the plates. Various numerical examples were considered and solved with the mixed FE-DQ method. The obtained results have been compared with the other analytical and numerical solutions and the validity of the mixed method has been justified. Also, it can be concluded that the FE mesh-sizes have more influence in convergence than DQ nodal density. Using the ability of present mixed FE-DQ method for analyzing the problems with complicated boundary conditions, the dynamic response and stability of plates partially resting on elastic foundation is modeled. The effects of elastic foundation area $\left(l_{x} \times l_{y}\right)$ on fundamental frequencies and critical buckling load of thick plates have investigated. As the elastic foundation area increase, the fundamental frequencies and critical buckling loads of the plates are found to be raised. The proposed method (FE-DQ) enjoys both the ability of FEM in modeling the complicated geometry and the simplicity and accuracy of DQM. The presented method can be extended to three-dimensional elasto-static and elasto-dynamic analysis of homogeneous, composite and functionally graded thick plates with complicated geometry with or without elastic foundation.

\section{Acknowledgement}

The authors would like to thank the anonymous referees for constructive comments on earlier version of this paper.

\section{References}

Bellman, R., Kashef, B. G., \& Casti, J. (1972). Differential quadrature: a technique for the rapid solution of nonlinear partial differential equations.Journal of computational physics, 10(1), 40-52. 
Bert, C. W., \& Malik, M. (1996). Differential quadrature method in computational mechanics: a review. Applied Mechanics Reviews, 49(1), 1-28.

Dehghan, M., \& Baradaran, G. H. (2011). Buckling and free vibration analysis of thick rectangular plates resting on elastic foundation using mixed finite element and differential quadrature method. Applied Mathematics and Computation, 218(6), 2772-2784.

Du, H., Lim, M. K., \& Lin, R. M. (1994). Application of generalized differential quadrature method to structural problems. International Journal for Numerical Methods in Engineering, 37(11), 18811896.

Gupta, U., Sharma, S., \& Singhal, P. (2016). DQM modeling of rectangular plate resting on two parameter foundation. Engineering Solid Mechanics,4(1), 33-44.

Lam, K. Y., Wang, C. M., \& He, X. Q. (2000). Canonical exact solutions for Levy-plates on twoparameter foundation using Green's functions.Engineering Structures, 22(4), 364-378.

Leissa, A. W. (1973). The free vibration of rectangular plates. Journal of Sound and vibration, 31(3), 257-293.

Liew, K. M., \& Teo, T. M. (1998). Modeling via differential quadrature method: three-dimensional solutions for rectangular plates. Computer Methods in Applied Mechanics and Engineering, 159(3), 369-381.

Liew, K. M., \& Teo, T. M. (1999). Three-dimensional vibration analysis of rectangular plates based on differential quadrature method. Journal of Sound and Vibration, 220(4), 577-599.

Lim, C. W. (1999). Three-dimensional vibration analysis of a cantilevered parallelepiped: exact and approximate solutions. The Journal of the Acoustical Society of America, 106(6), 3375-3383.

Matsunaga, H. (2000). Vibration and stability of thick plates on elastic foundations. Journal of engineering mechanics, 126(1), 27-34.

Mindlin, R. D. (1951). Influence of rotary inertia and shear on flexural motions of isotropic elastic plates. ASME Journal of Applied Mechanics, 18, 1031-1036.

Motaghian, S., Mofid, M., \& Akin, J. E. (2012). On the free vibration response of rectangular plates, partially supported on elastic foundation.Applied Mathematical Modelling, 36(9), 4473-4482.

Omurtag, M. H., Ozutok, A. \& Akoz, A. (1997). Free vibration analysis of Kirchhoff plates resting on elastic foundation by mixed finite element formulation based on Gateaux differential. International Journal for Numerical Methods in Engineering, 40(2), 295-317.

Pan, B., Li, R., Su, Y., Wang, B., \& Zhong, Y. (2013). Analytical bending solutions of clamped rectangular thin plates resting on elastic foundations by the symplectic superposition method. Applied Mathematics Letters, 26(3), 355-361.

Pasternak, P. (1954). On a new method of analysis of an elastic foundation by means of two foundation constants (in Russian). Gosudarstrennoe Izdatelstvo Literaturi po Stroitelstvu i Arkhitekture, Moscow, USSR.

Samaei, A. T., Aliha, M. R. M., \& Mirsayar, M. M. (2015). Frequency analysis of a graphene sheet embedded in an elastic medium with consideration of small scale. Materials Physics and Mechanics, $22,125-135$.

Takahashi, K. \& Sonoda, T. (1992). Dynamic stability of a rectangular plate on Pasternak foundation subjected to sinusoidally time-varying in-plane load. Theoretical and Applied Mechanics, 7, 55-62.

Teo, T. M., \& Liew, K. M. (1999). A differential quadrature procedure for three-dimensional buckling analysis of rectangular plates. International journal of solids and structures, 36(8), 1149-1168.

Thai, H. T. \& Choi, D.H. (2014). Zeroth-order shear deformation theory for functionally graded plates resting on elastic foundation. International Journal of Mechanical Sciences, 78, 35-43.

Thai, H. T., Park, M. \& Choi, D. H. (2013). A simple refined theory for bending, buckling, and vibration of thick plates resting on elastic foundation. International Journal of Mechanical Sciences, 73, 4052.

Timoshenko, S. P. \& Woinowsky-Krieger, W. (1970). Theory of Plates and Shells. New York: McGraw-Hill.

Ventsel, E. (2001). Thin plates and shells: Theory, Analysis, and Applications. Marcel Dekker Incorporated, New York, USA. 
Vimal, J., Srivastava, R., Bhatt, A., \& Sharma, A. (2014). Free vibration analysis of moderately thick functionally graded skew plates. Engineering Solid Mechanics, 2(3), 229-238.

Winkler, E. (1867). Die Lehre von der Elasticitaet und Festigkeit. Prag, Dominicus.

Xiang, Y. (2003). Vibration of rectangular Mindlin plates resting on non-homogenous elastic foundations. International journal of mechanical sciences, 45(6), 1229-1244.

Xiang, Y., Wang, C. M., \& Kitipornchai, S. (1994). Exact vibration solution for initially stressed Mindlin plates on Pasternak foundations. International journal of mechanical sciences, 36(4), 311 316.

Zhou, D., Cheung, Y. K., Lo, S. H., \& Au, F. T. K. (2004). Three-dimensional vibration analysis of rectangular thick plates on Pasternak foundation.International journal for numerical methods in engineering, 59(10), 1313-1334. 